\title{
Containment of a traceable COVID-19 outbreak among healthcare workers at a hematopoietic stem cell transplantation unit
}

\author{
N. Buchtele ${ }^{1}$ W. Rabitsch ${ }^{1} \cdot$ H. A. Knaus ${ }^{1} \cdot$ P. Wohlfarth ${ }^{1}{ }^{1}$
}

Received: 18 April 2020 / Accepted: 26 May 2020 / Published online: 1 June 2020

(C) Springer Nature Limited 2020

\section{To the Editor:}

SARS-CoV-2 and the associated disease COVID-19 have emerged as a pandemic with a rapid rise of cases across the world. Its spread has put an enormous pressure on healthcare systems in several countries, and an increasing number of healthcare workers are getting infected. In this correspondence, we report on a traceable outbreak of COVID-19 among healthcare workers at the Hematopoietic Stem Cell Transplantation Unit of the Medical University of Vienna and measurements taken for its successful containment.

Our Unit has 18 single bedrooms, of which eight are equipped with high efficiency particulate air (HEPA) filter positive pressure lock systems reserved for allogeneic hematopoietic stem cell transplant (HSCT) recipients. The remainder ten beds are placed in single-patient rooms with ambient air and are primarily used for autologous HSCTs or chimeric antigen receptor (CAR) T-cell therapies. By standard operating procedures (SOPs), all healthcare workers are obliged to wear surgical masks before entering a patient room and to wear gloves as well as disposable aprons before any direct patient contact. Patients undergoing HSCT are not allowed to leave their room during the phase of aplasia. With the beginning of the SARS-CoV-2 pandemic all other nonaplastic patients were also encouraged to stay in their patient rooms and no visitors were allowed. The HEPA-positive pressure systems were kept in operation, as they can be regulated individually for each patient room and could have been turned off in the case of required isolation. According to our staffing schedule, two physicians (out of a team of ten people) and five nurses (out of a team of 43 people) provide patient care during

P. Wohlfarth

philipp.wohlfarth@meduniwien.ac.at

1 Hematopoietic Stem Cell Transplantation Unit, Department of Medicine I, Medical University of Vienna, Vienna, Austria daytime, and one physician and three nurses during nighttime, respectively.

On March 23, a nurse working at our Unit was tested positive for SARS-CoV-2 detected by RT-PCR analysis of a nasopharyngeal swab. His symptoms included fever and dry cough that had developed during his nightshift. Six days previous to this event, he had tested negatively for SARSCoV-2 in the context of minor flu-like symptoms without fever or cough after having returned from a 3-week leave on the day before, and he therefore continued to work.

Our in-house SOPs required testing staff for SARS-CoV-2 who had direct contact with the index person for longer than $15 \mathrm{~min}$ or had unprotected contact with infectious secretion of the index person within the last 2 days before indicative symptoms developed. However, due to the highly vulnerable nature of our patients, we decided to screen all healthcare workers and cleaning personnel, who had any face-to-face contact regardless of length of exposure and extended the screening timeframe to the day of the index person's return from leave, having assumed a falsely negative PCR at the time of onset of flu-like symptoms. In addition, all patients hospitalized at our ward as well as those discharged within the screening timeframe were subjected to SARS-CoV-2 testing as described below.

After release of the positive PCR, immediate contact tracing allowed the identification of 23 healthcare workers (5 physicians; 18 nurses) and 3 persons from the cleaning staff who have had face-to-face contact to the index person. All identified persons were instantly subjected to RT-PCR from nasopharyngeal swabs. Swabs of three nurses turned out positive on the same day. As they were in an overall well condition with minimal to none evident symptoms, respective nurses were immediately subjected to a 14-day home quarantine.

The remainder healthcare personnel was allowed to continue their work as long as they had negative daily swabs for SARS-CoV-2, a mask covering mouth and nose with no vent (FFP-1) was worn at all times and no symptoms indicative for COVID-19. Personnel were encouraged to enhance hand 
hygiene and social distancing at work. Over the following 10 days of daily testing in healthcare workers who continued their work, two additional nurses tested positive for SARSCoV-2 and put under home quarantine for 14 days. Both nurses had developed symptoms prior to their positive test result and therefore were tested at home.

During the reported 4-week period between March 23 and the April 17, a total of 11 patients received a hematopoietic stem cell transplantation (allogeneic, $n=9$; autologous, $n=$ 2) and 25 additional patients, including 3 patients who received a CAR T-cell infusion, received inpatient treatment at our HSCT unit. All patients who had been discharged within the screening timeframe were tested for SARS-CoV-2. Discharged patients who tested negative were urged to undergo a 14-day home quarantine and regular daily telephone visits were performed. Inpatients were tested on every other day for SARS-CoV-2 or as soon as any symptoms indicative of COVID-19 developed. By April 17, all tests remained negative and no case of COVID-19 was detected among patients.

In summary, we report a traceable COVID-19 outbreak affecting five nurses at an HSCT unit. By choosing a strategy of extended contact tracing, broad testing, and strict hygiene measures we were able to prevent a further spread of the infection among personnel and its transmission to patients. We think that our experience highlights several issues, most of which have also been addressed by the recent EBMT recommendations on COVID-19 [1]:

(1) A negative test for SARS-CoV-2 from nasal swabs does not preclude an infection in people with atypical symptoms such as sneezing, general malaise, or others. Further, RT-PCR assays have been reported to have limited sensitivity for detecting SARS-CoV-2 infections, especially during the early phase of infection [2,3]. Hence, staff who experiences any systemic or respiratory symptoms of infection should stay at home and not go to work.

(2) HSCT recipients constitute a highly specialized patient population. Hence, several immediate measures in response to a COVID-19 outbreak practiced by other specialties, such as shutting-down a ward or transferring patients to other services, may not be feasible in the context of HSCT patients. Therefore, contingency plans should be prepared and ready at hand for when an outbreak of COVID-19 emerges in an HSCT service. Limiting the service to urgent transplants may preemptively reduce the risk of overstressing personnel resources in the context of an emergent outbreak.
(3) Facilitating measures of hygiene, establishing testing procedures, isolation of affected individuals, and social distancing at the work place require training, stringent information, and a strong dedication of all the staff working at an HSCT service. We believe that the familiarity of HSCT providers with the care of highly vulnerable immunocompromised patients serves as a great strength for us and our patients during the current crisis. In this context, we have observed a high amount of willingness and capability of all HSCT staff to adhere to the proposed measures. Nevertheless, great attention must be paid to the psychosocial consequences of these stressful working conditions. Staff should be supported by the establishment of means to address situations of psychosocial distress, such as telephone hotlines or support groups.

Prevention of infection with SARS-CoV-2 should be of utmost priority in HSCT recipients. COVID-19 cases among healthcare workers are increasing, including those working at HSCT services. Therefore, successful contact tracing remains a foundation of containment of COVID-19 spread especially among healthcare personnel. Our experience shows that thorough precaution and strict hygiene guidelines allow the continuation of routine care of patients at a HSCT unit and a successful containment of a local COVID-19 outbreak.

\section{Compliance with ethical standards}

Conflict of interest The authors declare that they have no conflict of interest.

Publisher's note Springer Nature remains neutral with regard to jurisdictional claims in published maps and institutional affiliations.

\section{References}

1. Ljungman $\mathrm{P}$, Mikulska $\mathrm{M}$, de la Camara R, Basak GW, Chabannon C, Corbacioglu S, et al. The challenge of COVID-19 and hematopoietic cell transplantation; EBMT recommendations for management of hematopoietic cell transplant recipients, their donors, and patients undergoing CAR T-cell therapy. Bone Marrow Transplant. 2020;13:1-6.

2. Ai T, Yang Z, Hou H, Zhan C, Chen C, Lv W, et al. Correlation of chest CT and RT-PCR testing in coronavirus disease 2019 (COVID-19) in China: a report of 1014 cases. Radiology. 2020: 200642. https://doi.org/10.1148/radiol.2020200642.

3. Fang Y, Zhang H, Xie J, Lin M, Ying L, Pang P, et al. Sensitivity of chest CT for COVID-19: comparison to RT-PCR. Radiology. 2020:200432. https://doi.org/10.1148/radiol.2020200432. 